\title{
Reflets
}

Revue ontaroise d'intervention sociale et communautaire

\section{Étude comparative des caractéristiques internes et de l'environnement externe des communautés d'entraide francophone et anglophone de Sudbury-Manitoulin}

\author{
Dyane Adam
}

Volume 1, numéro 2, automne 1995

La santé communautaire en Ontario français : défis et espoirs

URI : https://id.erudit.org/iderudit/026077ar

DOI : https://doi.org/10.7202/026077ar

Aller au sommaire du numéro

Éditeur(s)

Reflets : Revue ontaroise d'intervention sociale et communautaire

ISSN

1203-4576 (imprimé)

1712-8498 (numérique)

Découvrir la revue

Citer cet article

Adam, D. (1995). Étude comparative des caractéristiques internes et de l'environnement externe des communautés d'entraide francophone et anglophone de Sudbury-Manitoulin. Reflets, 1(2), 72-89.

https://doi.org/10.7202/026077ar
Résumé de l'article

La présente recherche porte sur la communauté d'entraide du district de Sudbury-Manitoulin(Ontario), secteur caractérisé, entre autres, par le multiculturalisme et le bilinguisme. Plus précisément,cette étude constitue une analyse comparative des communautés d'entraide francophoneet anglophone au niveau du champ d'intérêt des groupes, de leur organisation interne et de lanature des liens qu'il maintiennent avec leur environnement externe. Les résultats suggèrent queles groupes d'entraide des deux communautés en question diffèrent non seulement au point devue du nombre et de la diversité, mais également, et de façon significative, dans la nature desproblèmes traités et dans la participation du secteur professionnel. Il semble également y avoir unparallèle étroit entre l'organisation du système formel de soins de santé et des services sociaux etle développement de l'entraide dans les deux groupes étudiés.
Tous droits réservés (C Reflets : Revue ontaroise d'intervention sociale et communautaire, 1995
Ce document est protégé par la loi sur le droit d'auteur. L'utilisation des services d'Érudit (y compris la reproduction) est assujettie à sa politique d'utilisation que vous pouvez consulter en ligne. 


\section{Étude comparative des caractéristiques internes et de l'environnement externe des communautés d'entraide francophone et anglophone de Sudbury-Manitoulin}

L a présente recherche porte sur la communauté d'entraide du distrid de Sudbury-M anitoulin (0 ntario), secteur caractérisé, entre autres, par le multiaulturalisme et le bilinguisme. Plus prédsément, cette étude constitue une analyse comparative des communautés d' entraide francophone et anglophone au niveau du champ d'intérêt des groupes, de leur organisation interne et de la nature des liens qu'il maintiennent avec leur environnement externe. L es résultats suggèrent que les groupes d'entraide des deux communautés en question diffèrent non seulement au point de vue du nombre et de la diversité, mais également, et de façon significative, dans la nature des problèmes traités et dans la partiapation du secteur professionnel. II semble également y avoir un parallèle étroit entre l'organisation du système formel de soins de santé et des services sociaux et le développement de l'entraide dans les deux groupes étudiés.

\section{$D$ yaneA dam}

P rofesseure et Principale, $C$ ollège universitaire de $G$ Iendon

\section{Introduction}

Au cours des dernières décennies, la diversité des groupes d'entraide (GE) ainsi que le nombre d'entraidants ont connu une expansion remarquable, à tel point qu'ils ont fini par représenter la ressource communautaire la plus importante pour faire face aux nombreux défis et épreuves qui jalonnent une vie humaine. Bien 
«... la recherche sur

l'entraide a largement passé sous silence la variable ethnoalturelle comme facteur déterminant.» que la littérature et les recherches décrivent généralement bien les mérites particuliers et les caractéristiques générales des GE (R omeder, 1982), le contexte dans lequel ils évoluent et l'influence du milieu ambiant sur les groupes n'ont pas encore été suffisamment étudiés (Levy, 1984; Leventhal, M aton et M adara, 1988).

D es recherches, en nombre limité, ont tenté d'étudier le rapport entre les GE et leur environnement externe; c'est-à-dire l'évolution des groupes, leurs caractéristiques, leurs besoins, leurs fonctions et leur efficacité par rapport aux communautés particulières dont ils émanent ( $M$ aton, Leventhal, $M$ adara et Julien, 1989; M aton, 1989). Selon Levy (1984), les GE constituent des systèmes sociaux miniatures dont les caractéristiques varient en fonction des systèmes sociaux plus larges dont ils font partie. C'est pourquoi le contexte socio-géographique d'insertion desGe (c'està-dire urbain, semi-urbain, rural), I'homogénéité culturelle de la communauté et l'appui accordé au groupe par les systèmes officiels constituent tous des variables qui affectent la survie du GE, ses caractéristiques générales et sa croissance.

Par contre, la recherche sur l'entraide a largement passé sous silence la variable ethno-culturelle comme facteur déterminant. O n dispose de peu d'information quant à la participation des groupes ethniques aux systèmes d'entraide répertoriés et étudiés (Gottlieb, 1983) si ce n'est pour constater que certaines minorités ethniques désavantagées des États- $\mathrm{U}$ nis ( $\mathrm{N}$ oirs, $\mathrm{H}$ ispanophones et A utochtones américains) se distinguent par le recours à d'autres formes d'entraide communautaire que celles privilégiées par la population américaine de race blanche (H amilton, 1980; 1990). D e même, au $C$ anada, il existe peu de tentatives de comparaison de la répartition des groupes d'entraide entre les trois communautés fondatrices du pays: la population autochtone (avec statut), la communauté d'expression française et la population de langue anglaise. Toutefois, une étude récente réalisée par St-Amand et Vuong (1994) a démontré des différences entre les populations anglophone et acadienne dans la nature du réseau d'aide sur lequel s'appuient les ex-psychiatrisés du N ouveau-Brunswick: le premier groupe privilégie l'appareil institutionnel tandis que le groupe minoritaire dépend davantage de son réseau social. 
«L a variable langue est largement occultée des recherches et des études qui inspirent aduellement les politiques ontariennes en matière de santé et services sodiaux, le français étant souvent confiné à la simple traduction de ce qui est conçu par et pour la population majoritairement anglophone»
De fait, la rareté des études empiriques sur la dimension socioculturelle des réseaux d'aide naturelle se retrouve également dans le réseau d'aide institutionnelle. Les variables culture et langue ont généralement été peu intégrées au niveau de la recherche et du développement despolitiques et pratiques en matière de santé et services sociaux, même dans les provinces les plus bilingues du C anada (Bibeau et al. , 1992; C orin et al., 1990; St-A mand etVuong, 1994). Plus près de nous, en 0 ntario, ce n'est que depuis les six dernières années que la minorité d'expression française est en droit d'exiger des services sociaux et de santé dans sa langue (Loi sur les services en français, proclamée en 1989). Si on en juge par les textes de référence publiés récemment par les ministères responsables de ces services (C onseil du premier ministre sur la santé, le bien-être et la justice sociale, 1994a, 1994b, 1994c; ministère de la Santé, 1993; ministère des Services sociaux et communautaires, 1987), force est de reconnaitre que cette législation a eu bien peu d'impact sur le cadre conceptuel de planification et de livraison des soins de santé et des services sociaux. La variable langue est largement occultée des recherches et des études qui inspirent actuellement les politiques ontariennes en matière de santé et services sociaux, le français étant souvent confiné à la simple traduction de ce qui est conçu par et pour la population majoritairement anglophone. Pourtant, les travaux empiriques d'orientation ethnographique d'une équipe de chercheurs québécois (Bibeau et al., 1992; C orin et al., 1990) remettent en question la pertinence du modèle épidémiologique dominant pour garantir l'adéquation socioculturelle des services dans un milieu donné. U $n$ tel modèle se caractérise par «e mouvement du haut vers le bas; les études de besoins des populations et celles de prévalence des problèmes sont les principaux outils utilisés pour établir les priorités»; enfin, leur «objectif implicite(... ) est de promouvoir l'uniformité et l'homogénéité plutôt que la différenciation et les spécificités» (Bibeau et al., 1992:251). C es mêmes auteurs déplorent le fait que «peu de travaux se sont systématiquement intéressés à la manière dont des dynamiques socio-communautaires orientent ou infléchissent la présence, la composition ou le fonctionnement des réseaux d'aide informelle dans des milieux particuliers» (Ibid:68). 
C'est dans une perspective d'analyse socio-culturelle des réseaux d'aide naturelle que se situe la présente recherche. $\mathrm{N}$ ous examinerons en particulier la croissance et les caractéristiques générales des GE oeuvrant dans le district de Sudbury- $M$ anitoulin, région du nord de l'O ntario centrée surtout sur l'exploitation de richesses naturelles et caractérisée, entre autres, par la dispersion démographique, le multiculturalisme et le bilinguisme.

\section{Description de la recherche}

Les données sur lesquelles se base cette recherche sont tirées d'une étude plus large des besoins et de la structure des groupes d'entraide dans le N ord-est ontarien réalisée en 1989 (A dam et Hoehne, 1990).

Cette étude avait pour but de fournir des données empiriques sur la nature et l'organisation du réseau d'entraide dans la région de Sudbury-M anitoulin en tenant compte particulièrement du contexte socio-linguistique dans lequel il se situe. En raison de l'espace limité dont nous disposons, nous restreindrons notre analyse à trois jeux de variables significatives pour illustrer certaines différences dans les conditions de vie des groupes: problèmes ou champ d'intérêt qui leur sont particuliers, environnement interne (caractéristiquesinternes) et environnement externe ( $M$ aton, 1989).

\section{Population cible}

U n aperçu général descaractéristiques socio-démographiques de la région de Sudbury- $M$ anitoulin servira de toile de fond à notre analyse comparative du secteur de l'entraide dans les deux principales communautés linguistiques, les Anglophones et les Francophones.

Le D istrict de Sudbury-M anitoulin s'étend sur environ 50000 kilomètres carrés dans le N ord-est de l'O ntario; environ le quart de sa population (200 000 personnes) est dispersé sur un vaste 
«L a grande majorité des $F$ rancophones de Sudbury-M anitoulin $(96 \%)$ parle couramment l'anglais, bien qu' environ les trois quarts utilisent encore le français comme langue de communication au foyer.» territoire. La principale activité économique est générée par l'exploitation des ressources minières. Étant donné que la municipalité régionale de Sudbury représente plus de $80 \%$ de la population régionale et fait office de centre d'accès à une gamme importante de services publics, on la qualifie souvent de métropole du nord de l'O ntario. À bien des égards, mais principalement à cause de sa dispersion démographique et de ses particularités bilingues et multiculturelles, cette région constitue un microcosme du $C$ anada. Les groupes ethniques, fondateurs de l'ensemble du C anada, se répartissent ainsi dans la région: $32,6 \%$ de langue anglaise, 33,8 \% de langue française et $3 \%$ de gens avec statut amérindien; le dernier tiers de la population comprend diverses communautés d'immigrants (Italiens, U krainiens, Allemands, etc.) qui ont adopté, en grande majorité, l'anglais comme langue d'usage. Ils forment ainsi avec la population d'expression anglaise de la région le groupe linguistique dominant dans tous les secteurs des services et de la vie communautaire.

De plus, les deux communautés minoritaires (francophones et autochtones) sont particulièrement désavantagées aux plans du revenu, de l'emploi et du niveau d'éducation. Ainsi, par rapport à leurs homologues anglophones et aux Francophones d'autres parties de la province, la population francophone du N ord de I'O ntario se trouve au bas de l'échelle en termes de revenu et d'éducation (Lavoie et St-Germain, 1991). O n y observe également un phénomène important d'acculturation (C lément et al, 1993). La grande majorité des Francophones de Sudbury$M$ anitoulin (96\%) parle couramment l'anglais, bien qu'environ les trois quarts utilisent encore le français comme langue de communication au foyer (H opkins, 1985).

\section{Collecte des données}

U n inventaire des groupes d'entraide de la région de Sudbury$M$ anitoulin a été réalisé à partir des répertoires existants et des renseignements fournis par des informateurs clés. Le concept de groupe d'entraide utilisé dans cette étude a été défini en utilisant les critères suivants: les membres partagent le même problème 
ou les mêmes préoccupations, les membres dirigent eux-mêmes leur groupe avec une intervention minimale de personnes professionnelles ou qui ne sont pas touchées par la problèmatique; le groupe n'a pas de but lucratif et la participation est volontaire; et finalement, il se caractérise par des activités d'échange mutuel.

\section{Le questionnaire}

U n questionnaire a servi à recueillir des données sur les aspects suivants des GE: les caractéristiques organisationnelles, les questions ou problèmes traités, les types de services, I'interaction avec d'autres groupes et/ ou organismes, les sources de financement, les principaux défis et besoins relevés. $C$ et outil a été conçu de manière à répondre à l'objectif visé au départ et à fournir des données faciles à coder. Comportant 64 questions, il s'inspire d'une version similaire utilisée dans une recherche semblable menée au M anitoba (H oehne, 1987) et se divise en deux parties, I'une constituée de questions à choix multiples et l'autre de questions ouvertes.

À l'origine, le questionnaire a été envoyé à $115 \mathrm{GE}$; il était accompagné d'une lettre décrivant l'objectif de la recherche et indiquant que l'on communiquerait par téléphone avec la personne ressource pour obtenir verbalement les réponses à certaines questions, notamment les questions ouvertes. De fait, ce contact téléphonique a permis non seulement de recueillir de vive voix des réponses à certaines questions à caractère plus qual itatif, mais a également entraîné un meilleur taux de réponse.

\section{Analyse des résultats}

Des 115 questionnaires envoyés, 74 ont été reçus, soit un taux de réponse de $64 \%$. Un formulaire a dû être éliminé car l'organisme ne répondait pas aux critères de groupe d'entraide mentionnés ci-haut. 
En ce qui concerne la langue de communication du groupe, I'anglaisa été utilisé par la plupart des personnes qui ont répondu au nom du groupe (73\%). Seulement $23 \%$ des groupes fonctionnent en français et un seul utilise occasionnellement la langue amérindienne, même si deux groupesd'expression anglaise s'adressent spécifiquement à desA utochtones (4\%). Environ une douzaine de groupesanglophones ont déclaré accueillir des membres francophones qui, de temps en temps, communiquent en français entre eux. La plupart des groupes (93\%) sont basés dans la région de Sudbury; les GE dans les collectivités rurales isolées représentent $6,5 \%$ des répondants. M ême si un certain nombre de Francophones réside dans des villages autour de Sudbury ( $H$ anmer, $R$ ayside- $B$ alfour, etc.), nousn'avons pas relevé de différence significative entre les groupes anglophones et francophones au niveau de cette variable.

D es comparaisons entre les groupes, en tenant compte du facteur langue, ont été effectuées à l'aide de recoupements statistiques et de mesures de chi-carré. Le petit nombre de groupes autochtones $(n=3)$ restreint I'utilisation de tests statistiques. Les comparaisons qui suivent ne portent donc que sur les groupes anglophones et francophones.

\section{Problèmes et champs d'action particuliers}

Les ge participant à l'étude devaient identifier parmi un large éventail de possibilités de réponses les champsd'action et les problèmes particuliers sur lesquels ils se concentraient. Les réponses ont été regroupées autour de trois thèmes: problèmes de santé (ex. maladie chronique, naissance, santé mentale, toxicomanie, etc.); problèmes sociaux et action sociale (ex. mauvais traitements infligés aux enfants, victimes de crimes, logement, pauvreté, violence, alcoolisme, etc.); et croissance personnelle/ problèmes d'éducation (ex: analphabétisme, difficultés d'apprentissage, aptitudes à la communication, éveil spirituel, etc.).

$N$ ous avons noté des différences importantes $(\underline{\alpha}=0,001)$ entre les groupes francophones et anglophones au niveau de la problématique privilégiée par les groupes. À une exception près, 
la communauté francophone ne s'est pas dotée de groupes axés sur la santé. Par contre, dans la communauté d'expression anglaise, la majorité des groupes $(50,9 \%)$ sont axés sur de telles questions. LeSGE francophones se répartissent entre la catégorie des problèmes sociaux (47\%) et celle de la croissance personnelle/ éducation (53\%). En comparaison, un pourcentage important des groupes anglophones $(41,5 \%)$ se retrouve dans la catégorie des problèmes sociaux. Seulement $7 \%$ des groupes anglophones s'intéressent aux questions de croissance personnelle et d'éducation. $U$ ne analyse plus poussée a démontré qu'une proportion importante des groupes francophones actifs dans cette dernière catégor ie ont en fait comme thème principal l'éveil et la croissance spirituelle. On peut en déduire que les champs d'intérêt desgroupes d'entraide francophones diffèrent largement des préoccupations des groupes de langue anglaise.

\section{Nature des services}

LeT ableau 1 énumère la liste des différents services offerts par les GE de chaque catégorie. $C$ eux-ci offrent plusieurstypes de services à leurs membres, dont le soutien (78\%), l'éducation/information (69\%), la consultation/ liaison (43\%), I'aiguillage vers d'autres services (42\%) et la croissance personnelle/ adaptation (37\%). Parmi les services mentionnés moins fréquemment, on retrouve l'assistance et la thérapie (22\%), les services physiques (17 \%) et les services matériels (15\%). Ces données coïncident avec de nombreuses observations déjà rapportées dans la littérature sur le rôle distinct desgroupes d'entraide comme ressource complémentaire aux systèmes officiels de santé et de services sociaux centrés surtout sur la thérapie et l'assistance.

II existe des différences importantes dans le type de services offerts par les groupes anglophones et francophones. En effet, dans six des quatorze catégories énumérées dans le tableau 1, ces deux groupes se distinguent dans les domaines suivants: soutien, éducation, consultation, aiguillage vers d'autres services, croissance personnelle et spirituelle. À l'exception de cette dernière catégorie, les groupes francophones ont déclaré être moins engagés 
dans les services de soutien et dans les activités d'éducation et d'information. C es derniers font également peu de consultation et d'aiguillage vers d'autres services.

Tableau 1

Services offerts par les GE et proportion des groupes actifs dans chaque catégorie

Type de service $\%$ de GE

\begin{tabular}{lcccc} 
& \multicolumn{2}{c}{ Anglais } & Français & Total \\
& $\mathbf{n = 5 4}$ & $\mathbf{n = 1 7}$ & $\mathbf{n - 7 4}$ & $\alpha$ \\
\hline A ppui & 87,0 & 47,2 & 77,8 & $\underline{0,00}$ \\
Éducation/information & 81,5 & 41,2 & 69,4 & $\underline{0,00}$ \\
Consultation/liaison & 51,9 & 11,8 & 43,1 & $\underline{0,00}$ \\
Aiguillage & 48,1 & 17,6 & 41,7 & $\underline{0,03}$ \\
Thérapie & 18,5 & 29,0 & 22,2 & n.s. \\
Croissance personnelle & 40,7 & 11,8 & 37,5 & $\underline{0,03}$ \\
Croissance spirituelle & 13,0 & 70,6 & 29,2 & $\underline{0,00}$ \\
Protection des droits & 25,9 & 5,9 & 19,4 & n.s. \\
(action politique) & & & & \\
Assistance physique & 16,7 & 11,8 & 16,7 & n.s. \\
A ssistance matérielle & 18,5 & 11,8 & 15,3 & n.S. \\
Activités de loisir & 33,3 & 35,3 & 34,7 & n.s. \\
Vie quotidienne & 18,5 & 3,5 & 15,3 & n.s. \\
A daptation personnelle/ & 37,5 & 42,6 & 23,5 & n.s. \\
sociale & & & & \\
Divers & 1,4 & 0,0 & 5,9 & n.s. \\
\hline
\end{tabular}

\section{Organisation interne}

N ousn'avons pas relevé de différences significatives entre les deux groupes linguistiques en ce qui touche les mécanismes d'organisation interne, définis par l'âge du groupe, les formes d'organisation, les critères d'admission des membres, lesstructures de réunion et les processus de prise de décision. En bref, les résultats démontrent que les communautés d'entraide des districts de Sudbury$M$ anitoulin se comparent, au niveau organisationnel, à ce qui se fait ailleurs en A mérique du $\mathrm{N}$ ord (R omeder, 1982). 


\section{Affiliations et liens externes}

Plusieurs GE maintiennent des relations avec le système de soins formels en faisant régulièrement appel à des services professionnels pour des consultations (44,4\%), de l'aide occasionnelle (40,3\%), de la thérapie $(18,1 \%)$ ou à titre d'observateur $(20,8 \%)$. D ans d'autres cas, ces invités font partie de conseils ou de comités et agissent comme personnes-ressources. C ette coopération avec le secteur professionnel correspond à une croyance répandue (69\%) chez les Ge que ceux-ci constituent un complément aux services professionnels et qu'ils accordent beaucoup d'importance $(80,1 \%)$ au fait d'obtenir l'appui du réseau professionnel.

Par ailleurs, la collaboration entre la communauté d'entraide et le système officiel d'aide varie considérablement entre les deux groupes linguistiques. A lors que pour $27,8 \%$ des groupes anglophones, l'initiative de leur création vient de professionnels, c'est le cas seulement pour $5,9 \%$ du côté francophone $(M=3,56, d f=1$, $\underline{\alpha}=0,05)$. Le personnel professionnel semble donc jouer un rôle marginal dans ce dernier cas et important dans le premier. Du côté francophone, ces personnes agissent comme observateurs dans $47 \%$ des cas, par rapport à seulement $13 \%$ dans les groupes anglophones $(M=9,02, \underline{d f}=1, \underline{\alpha}=0,003)$. Inversement, $59,3 \%$ des groupes anglophones font appel au personnel professionnel pour des conseils, par rapport à seulement 5,9\% du côté francophone ( $M=14,8, \underline{d f}=1, \underline{\alpha}=0)$. C es liens restreints avec le système formel de soins se confirment également par le fait que peu de groupes francophones $(11,8 \%)$ peuvent être contactés par le biais d'organismes officiels, démarche courante $(75,9 \%)$ dans les réseaux anglophones $(M=22,2, \underline{d f}=1, \underline{\alpha}=0)$.

O n peut aussi évaluer le type de liens que maintiennent les GE avec leur environnement externe en examinant l'accès du groupe aux structures de soutien communautaires telles que des salles de réunions et des services de téléphone et d'aiguillage. Plus des trois quarts des GE utilisent des salles de réunion dans la communauté, c'est-à-dire dans les hôpitaux (17,1\%), les organismes de service social $(17,1 \%)$, les églises $(14,3 \%)$, les organismes de charité $(8,6 \%)$, les écoles $(7,6 \%) ; 7,6 \%$ ont leurs propres salles. 
Environ $25 \%$ doivent compter sur leurs propres ressources pour répondre à leurs besoins d'espace. Seulement 18,1 \% disposent de leur propre numéro de téléphone; pour les autres $(66,7 \%)$, les membreséventuels ou toute autre personne doivent appeler chez un particulier faisant partie du groupe en question.

Figure 1

\section{Moyens d'accès au groups}

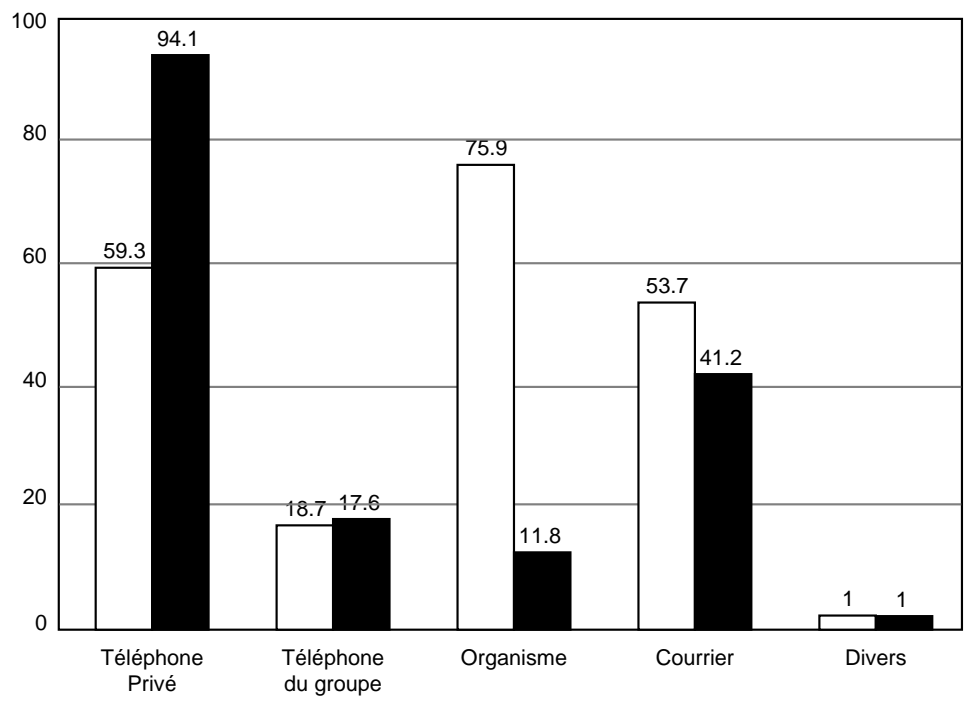

GE anglophones

GE francophones

II existe cependant un écart important entre les deux groupes linguistiques au niveau du soutien reçu de la part de l'appareil institutionnel. Comme l'illustre la figure 1 , presque tous les GE francophones $(94,1 \%)$ dépendent de la bonne volonté d'un membre qui donne son numéro de téléphone personnel que les personnes intéressées peuvent composer, alors que c'est le cas seulement pour $59,3 \%$ des groupesanglophones $(M=7,17, \underline{d f}=2$, $\underline{\alpha}=0,02)$. D e plus, alors que $31,5 \%$ des groupes anglophones ont 
accès à des salles de réunion dans des organismes de services sociaux et 35,2 \% à des locaux dans des hôpitaux, aucun GE francophone ne rapporte l'utilisation de telles ressources institutionnelles (respectivement $M=7,04, \underline{d f}=1, \underline{\alpha}=0,007$ et $M=8,17, \underline{d f}=1$, $\underline{\alpha}=0,003)$. Pour $41,5 \%$ des groupes francophones, les réunions se tiennent principalement dans les églises $(M=4,45, \underline{\alpha}=0,03)$ et pour 29,4\%, chez des particuliers (Figure 2 ).

Figure 2

\section{Structure de soutien à la disposition des groupes d'entraide}

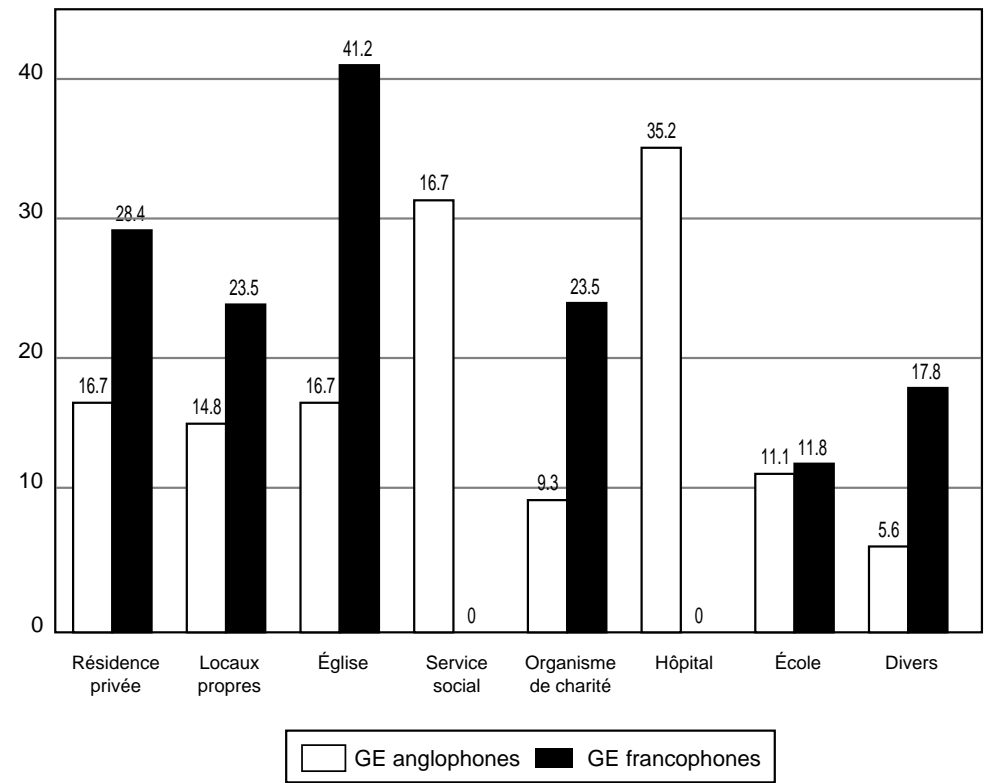

II existe cependant un écart important entre les deux groupes linguistiques au niveau du soutien reçu de la part de l'appareil institutionnel. C omme l'illustre la figure 1, presque tous les GE francophones $(94,1 \%)$ dépendent de la bonne volonté d'un membre qui donne son numéro de téléphone personnel que les personnes 
intéressées peuvent composer, alors que c'est le cas seulement pour $59,3 \%$ des groupes anglophones $(M=7,17, \underline{d f}=2, \underline{\alpha}=0,02)$. De plus, alors que $31,5 \%$ des groupes anglophones ont accès à des salles de réunion dans des organismes de services sociaux et $35,2 \%$ à des locaux dans des hôpitaux, aucun GE francophone ne rapporte I'utilisation de telles ressources institutionnelles (respectivement $M=7,04, \underline{d f}=1, \underline{\alpha}=0,007$ et $M=8,17, \underline{d f}=1, \underline{\alpha}=0,003)$. Pour $41,5 \%$ des groupes francophones, les réunions se tiennent principalement dans les églises $(M=4,45, \underline{\alpha}=0,03)$ et pour $29,4 \%$, chez des particuliers (Figure 2 ).

\section{Discussion et conclusion}

«E n tenant compte $d u$ facteur langue, nous avons observé des différences notables dans la nature et I'organisation des communautés d'entraide de SudburyM anitoulin.»
En tenant compte du facteur langue, nous avons observé des différences notables dans la nature et l'organisation des communautés d'entraide de Sudbury- $M$ anitoulin. N otre recherche démontre que les groupes existants sont non seulement concentrés dans la municipalité de Sudbury mais fonctionnent aussi presque exclusivement en anglais, en dépit du fait que les Francophones représentent plus du tiers de la population du district de Sudbury. $\mathrm{N}$ ous avons remarqué en particulier que les types de GE varient considérablement d'un groupe linguistique à l'autre. La plupart des GE francophones s'occupent de la croissance personnelle et spirituelle alors que la majorité des groupes anglophones sont axés sur la santé. De plus, les GE anglophones maintiennent un lien plus étroit avec le réseau institutionnel, y puisant appuis matériels (ex. salles de réunion, ligne téléphonique, etc.) et humains (ex. personne- ressource, consultation, etc.) ainsi qu'y référant au besoin certains de leurs membres. $U$ ne analyse sommaire du dernier $G$ uide des groupes $d^{\prime}$ entraide de Sudbury-M anitoulin (1993) suggère que ces tendances entre les $G E$ francophones et anglophones se maintiennent au moins à deux niveaux: aucun GE axé sur la croissance spirituelle ne fonctionne en anglais, et sur les 58 GE répertoriés en santé mentale et physique, seulement 6 sont d'expression française. 
«... la quasi-absence d'une infrastructure formelle dans I'organisation des soins de santé en français et le manque d'un réseau de soutien communautaire.»
Parmi les causes possibles de telles différences, mentionnons la quasi-absence d'une infrastructure formelle dans l'organisation des soins de santé en français et le manque d'un réseau de soutien communautaire. Les organismes de parrainage des $\mathrm{GE}$ actuels dans le secteur de la santé, comme l'A ssociation canadienne pour la santé mentale, la Société canadienne du cancer et la Société d'Alzheimer, fonctionnent presque entièrement en anglais. II est important de souligner que la plupart des GE qui s'adressent à des minorités bénéficient de l'aide financière d'organismesnationaux ou internationaux tels queA IcooliquesA nonymes, ou d'organismes sociaux reconnus par les Francophones et les A utochtones. Par exemple, les GE autochtones (Fourwinds, $\mathrm{N}$ ative Tenant A ssociation et un groupe axé sur la toxicomanie) sont tous associés au N 'Swakamok N ative Friendship Centre de Sudbury. C e centre aide la population autochtone à soutenir et à assurer la viabilité desquelques groupes d'entrai de conçusà leur intention. D es résultats similaires ont été rapportés ailleurs pour les minorités noires et hispaniques des États $U$ nis. $\mathrm{H}$ amilton (1980) indique que les sections noires et hispaniques de ParentsA nonymes, contrai rement aux sections anglophones (qui ont été mises sur pied avec l'aide de professionnels ou de parents volontaires), ont débuté grâce à I'aide d'une organisation nationale ou d'un programme universitaire d'action sociale. Ces données confirment d'autres études dansce domaine qui démontrent que les GE augmentent en nombre et en efficacité lorsqu'ils sont appuyés et soutenus par une infrastructure quelconque ( $M$ aton et al, 1989), particulièrement les centres d'information sur l'entraide.

D ans le secteur de la santé, aucun organisme ou agence ne sadressait uniquement aux besoins de la communauté francoontarienne de Sudbury- $M$ anitoulin. $U$ ne des conséquences importantes de la L oi ontarienne sur les services en français (1986) a été la création depuis les dernières années d'une demi dizaine de centres de santé communautaire francophones en province, dont un qui a tout récemment ouvert ses portes dans le district de Sudbury-M anitoulin. II sera intéressant, dans des études subséquentes, de noter l'impact de ce nouvel établissement de santé francophone sur l'évolution du secteur de l'entraide dans la 
«L e fait que la plupart des $G$ E francophones soient centrés sur la croissance spirituelle et qu'ils se réunissent dans les locaux des églises suggère que la paroisse joue encore un rôle dé en matière d'appui et de réseautage dans la communauté francophone» communauté francophone et de savoir plus particulièrement si les GE centrés sur la santé connaîtront une expansion parallèle. En se basant sur les expériences et études mentionnées précédemment, il y a lieu de s'attendre à un essor des GE au service de la communauté minoritaire de langue française dans ce secteur. U ne telle démonstration devient particulièrement importante dans le contexte actuel où les services sociaux et de santé sont soumis à d'énormes compressions budgétaires; tous savent déjà que la communautarisation et le souci d'efficience des services seront au centre des réformes gouvernementales du gouvernement $\mathrm{H}$ arris.

Le fait que la plupart des GE francophones soient centrés sur la croissance spirituelle et qu'ils se réunissent dans les locaux des églises suggère que la paroisse joue encore un rôle clé en matière d'appui et de réseautage dans la communauté francophone. À cet égard, la minorité francophone ressemble à d'autres minorités d'Amérique du N ord. Ainsi, au sein des communautés noires et hispaniques des États- $U$ nis, on fait souvent référence à l'église comme la source la plus commune d'aide extra-familiale en matière d'appui et de conseils au plan émotif (Gottlieb, 1983). II est également intéressant d'établir un parallèle entre les résultats de notre recherche et des observations faites par St-A mand etVuong (1994) dans un autre contexte minoritaire canadien. Leur étude démontre des différences entre les ex-psychiatrisés anglophones et francophones au niveau du réseau d'aide privilégié par chaque groupe, le réseau institutionnel étant plus important que le réseau social chez les Anglophones et inversement, pour les A cadiennes et A cadiens. U n fait fort intéressant à signaler dans cette étude est qu'ils ont observé que des considérations d'ordre spirituel - ce qu'ils ont appelé la dimension sacrée de l'aide - se retrouvent surtout chez lesA cadiennes et A cadienslorsqu'ilscommentent les pratiques professionnelles des soins.

O $n$ s'entend généralement pour reconnaitre que les cultures anglophone et francophone se sont construites autour d'une idéologie bien différente, l'une surtout de tradition protestante et l'autre de tradition catholique. A lors que la première favorisait 
«... c'est que toute planification des services véritablement adaptée à la minorité franco-ontarienne devra tenir compte des valeurs-pivots et de la dynamique sodiale particulières aux communautés locales» l'individualisme et la prise en charge de responsabilité personnelle, la seconde privilégiait l'autorité et la conscience du collectif. On pourrait émettre I'hypothèse qu'une des valeurs structurantes et différenciantes du tissu socio-communautaire de la minorité franco-ontarienne de Sudbury- $M$ anitoulin, et peut-être d'ailleurs en province et au pays, réside dans cette dimension religieuse. D'ailleurs, la survie et le développement de la communauté franco-ontarienne ont été, au fil de son histoire, intimement liés à l'Église; les écoles françaises et les quelques établissements de santé francophones étaient largement sous le contrôle des communautés religieuses (C arrière, 1994; Welch, 1995). D ans le secteur de la santé et des services sociaux, C arrière (1994) rapporte que la majorité de ces services sont offerts à la communauté franco- ontarienne par l'entremise d'agences et d'organismes unilingues anglophones. Se peut-il, comme le suggèrent StAmand et Vuong (1994) par rapport à la minorité acadienne, qu'à défaut de ressources institutionnelles et communautaires adaptées à leurs réal ités et à leurs spécificités, un groupe minoritaire «se voit contraint d'inventer ses réseaux, de créer des alternatives qui soient davantage à sa portée» (p.194)? U ne chose est certaine, c'est que toute planification des services véritablement adaptée à la minorité franco-ontarienne devra tenir compte des valeurs pivots et de la dynamique sociale particulières aux communautés locales. Sur ce sujet, I'O ntario français a un urgent besoin d'études tant épidémiologiques que sociologiques et anthropologiques pour documenter ses réalités et spécificités locales et régionales et inspirer adéquatement la planification et l'adaptation socio-culturelle des services sociaux et de santé dans l'ensemble de la province.

$\mathrm{N}$ ous remercions sincèrement toutes les personnes qui nous ont permiş, chaaune à sa façon, de mener cette recherche à bonne fin. $\mathrm{N}$ ous tenons à souligner la contribution des membres de groupes d' entraide qui ont bien voulu remplir le questionnaire et partiaper aux entrevues. $N$ ous exprimons aussi notre reconnaissance aux membres d'E ntraide $\mathrm{N}$ ord/ Self-H elp N orth/W eedookaaz owin G eewedin pour leurs judideuses 
Reflets

suggestions, leurs conseils et, le plus important de tout, leur appui moral. $\mathrm{N}$ ous adressons des remerdements particuliers à $\mathrm{K}$ aren $\mathrm{A}$ nnis, B everly $B$ lake, $D$ iane $B$ oucher, $R$ ichard $C$ arrière et $K$ ate T illiczek. $L$ isa $\mathrm{L} a \mathrm{~F}$ ramboise a effectué un travail remarquable au niveau de l'analyse informatisée et de la préparation de ce document. L'auteure souhaite remercier $M$. John L ewko et M me C ynthiaW hissel pour leurs précieux conseils sur la conception et l'analyse statistique du travail de recherche. E nfin, nous avons apprédé le soutien financier que nous ont accordé le ministère du $D$ éveloppement du $\mathrm{N}$ ord et le $\mathrm{F}$ onds de recherche de I' $\mathrm{U}$ niversité $L$ aurentienne

\section{Bibliographie}

ADAM , Dyane \& D ieter H OEH N E (1990). Structure and N eeds of Self-H elp G roups in the Sudbury$M$ anitoulin A rea, Sudbury, Presses de I'U niversité Laurentienne.

BIBEAU, Gilles, Alice CHAN -YIP, M argaret LOCK, Cécile RO U SSEAU, C arlo SERTLIN et $H$ ans FLEU RY (1992). L a santé mentale et ses visages: un Q uébec pluri-ethnique au quotidien, M ontréal, Gaëtan M orin éditeur.

CAR R IĖR E, R ichard (1994). «La Loi 8 et les services sociaux destinés aux familles francophones», C olloque F amilles francophones - M ultiples réalités Sudbury, 3-5 novembre.

CLÉMENT, R ichard, R enée GAUTHIER et Kimberley N O ELS (1993). «C hoix langagiers en milieu minoritaires: attitudes et identité concomitantes», $R$ evue canadienne des sciences du comportement, vol. 25, no 2, 149-164.

C O N SEIL DU PR EM IER M IN ISTRE (1994a). «La santé pour tous les O ntariens: dialogue sur les facteurs déterminants de la santé», Toronto, Presses de la $R$ eine.

C O N SEIL DU PR EM IER M IN ISTR E (1994b). «La décentralisation des services de santé et des services sociaux en $O$ ntario: R ecentrer le D ébat», Toronto: Presses de la $R$ eine.

C O N SEIL DU PR EM IER M IN ISTRE (1994C), «R ichesse et santé, santé et richesse», Toronto: Presses de la $R$ eine.

COR IN, H élène, Gilles BIBEAU, Jean-Claude MARTIN et R obert LAPLAN TE (1990). C omprendre pour soigner autrement, $M$ ontréal, Presses de I'U niversité de M ontréal.

GARTNER, Alan et Frank R IESSM AN (1977). Self-help in the human services, N ew York, Jossey Bass.

GOT T LIEB, B enjamin H . (1983). «O pportunitiesfor Collaboration with Informal Support Systems», dans S. C ooper \& W. H odges (eds.), T he F ields of M ental H ealth C onsultation, N ew York, H uman Sciences Press.

GOU VER NEM ENT DE L'ONTAR IO (1986). L oi ontarienne sur les services en français, Toronto, Presses de la $R$ eine.

H AM ILT O N,Alice (1980) «An Exploratory Study of T herapeutic Self-H elp C hild A buse Groups in Low-Income Populationss, LosAngeles: U niversity of C alifornia,T hèse de doctorat non publiée. 
H AM ILT O N, Alice (1990). «Self-H elp and M utual Aid in Ethnic M inority Communities», dans A.H. Katz \& E.I. Berder (eds), H elping $O$ ne A nother: Self-H elp $\mathrm{G}$ roups in a $\mathrm{C}$ hanging $W$ orld, California, Third Party Publishing.

H O EH N E, Dieter (1987). «Self-H elp Groups in M anitoba: G oals, Structures and N eeds», C ommunication présentée à la 3ième $C$ onférence nationale sur les politiques provinciales d'aide sodiale, Banff, Alberta.

H O EH N E, D ieter (1988). «Self-H elp and Social Change», dans F. Cunningham et al. (eds). Sodal $M$ ovements - Social $C$ hange: The Politics and Practice of $O$ rganizations,Toronto, B etween the Lines.

HO PKIN S, S.M . (1985). Social Indicator and D emographic D ata R eport for the D istrict of Sudbury and $M$ anitoulin, Sudbury, SSH R AG.

LAVO IE, M. et M. SAIN T-GER M AIN (1991). «Évolution comparative des revenus des Francoontariens), R evue du N ouvel-O ntario, 12.

LEVEN THAL, G.S., K.I. MATO N et Edward M ADAR A (1988). «Systemic O rganizational Support for Self-H elp Groups», A merican Journal of 0 rthopsychiatry, vol. 58, no 4, 592-603.

LEVY, Leon H . (1984). « ssues in R esearch and Evaluation», dansG artner,Alan et frank R IESSM AN (eds.), T he SelfH elp R evolution, $\mathrm{N}$ ew York, $\mathrm{H}$ uman Sciences Press.

M AT O N , K .I. (1989). «Towards an Ecological U nderstanding of M utual- H elp G roups:T he Social Ecology of Fit », A merican Journal of C ommunity Psychology, vol. 17, no 6, 729-752.

M AT O N , K.I., G.S. LEVEN TH AL, Edward J. M ADAR A et M. JU LIEN (1989). «FactorsAffecting the Birth and Death of M utual-H elp Groups: The R ole of $\mathrm{N}$ ational Affiliation, Professional Involvement, and M ember Focal Problem», A merican Journal of C ommunity Psychology, Vol. 17, no 5, 643-670.

M IN IST ĖR E DE LA SAN TÉ (1990). U ne meilleure santé pour l'0 ntario: progrès dans les années 1990, Toronto, Presses de la $R$ eine.

M IN IST ĖR E DES SERVICES SO CIAU X ET COM M U N AUTAIR ES (1987). D éfis et possibilités: $L$ 'intégration communautaires des personnes mentalement handicapées, Toronto, Presses de la $R$ eine.

RÉSEAU DE DÉVELOPEMENT DES GROUPES D'ENTRAIDE DE SUDBURYMAN ITO U LIN (1993). G uide des groupes d'entraide de Sudbury-M anitoulin/H andbook of Self$H$ elp and Support G roups, Sudbury, R éseau N ord.

R O M EDER, Jean M arie (1982). Self-H elp $G$ roups in $C$ anada. $O$ ttawa: $H$ ealth and Welfare $C$ anada. ST-AM AN D, N érée et D ungVU O N G (1994). «Q uand la langue fait une différence: ce que des“ bénéficiaires" pensent du système de santé mentale», Sodologie et sociétés, vol. 26, no 1, 179-196.

W ELCH , D avid (1995). «Les Franco- O ntariens: la résistance comme mode de vie», R eflets, vol. 1, no $1,20-42$. 